\title{
Modification of In-Hospital Recommendation and Prescription of Anticoagulants for Secondary Prevention of Stroke after Launch of Direct Oral Anticoagulants and Change of National Guidelines
}

\author{
Christian Gronemann Stephan Hause Anne Assmann Jens Neumann \\ Stefanie Schreiber Hans-Jochen Heinze Michael Goertler \\ Department of Neurology, Magdeburg University Vascular and Stroke Center, Magdeburg, Germany
}

\section{Keywords}

Stroke prevention - Direct oral anticoagulation - Atrial

fibrillation · Anticoagulant therapy · Guidelines

\begin{abstract}
Introduction: Approximately 1 out of 4 stroke patients suffers ischemic stroke secondary to atrial fibrillation (AF). Although indicated, withholding of anticoagulants for secondary prevention is a widespread phenomenon. Objective: We examined the longitudinal change of recommendation and prescription of secondary preventive anticoagulation in AF patients in an acute stroke center setting focusing on the impact of the introduction of direct oral anticoagulants (DOACs) and the change of national stroke prevention guidelines. Methods: Consecutive patients admitted with an acute cerebrovascular ischemic event underwent regular diagnostic work-up. Pseudonymized clinical data were entered into the institution's stroke registry. In those patients with AF, discharge letters were collected and evaluated for temporal trends and affecting factors of recommended and prescribed antithrombotic secondary medication at the
\end{abstract}

\begin{tabular}{ll}
\hline KARGER & ( ) 2020 The Author(s) Karger \\
& Published by S. Karger AG, Basel Open ciccess \\
karger@karger.com & This is an Open Access article licensed under the Creative Commons \\
www.karger.com/ced & $\begin{array}{l}\text { Attribution-NonCommercial-4.0 International License (CC BY-NC) } \\
\text { (http://www.karger.com/Services/OpenAccessLicense), applicable to } \\
\text { the online version of the article only. Usage and distribution for com- } \\
\text { mercial purposes requires written permission. }\end{array}$
\end{tabular}

time of discharge from hospital. Results: Of 7,175 patients admitted between January 2009 and December 2018, 1,812 (25.3\%) suffered stroke caused by AF. Frequency of patients with recommended anticoagulation increased within the observation period from 66.7 to $95.8 \%$ (per year; adjusted odds ratio $[\mathrm{OR}], 1.309$; confidence interval [Cl], 1.153-1.486). Independently from this time trend, DOAC approval (adjusted $\mathrm{OR}, 4.026 ; \mathrm{Cl} 1.962-8.265$ ) and guideline change (adjusted OR, 2.184; $\mathrm{Cl}, 1.006-4.743$ ) were associated with an increasing frequency of recommendation for anticoagulation. The rate of patients already receiving recommended anticoagulation for secondary prevention at discharge increased from 42.1 to $62.5 \%$. Introduction of DOACs was not associated with this trend, and guideline change was even associated with decreasing frequency of anticoagulated patients at hospital discharge (adjusted OR, 0.641; $\mathrm{Cl}, 0.414-0.991$ ). Fear of early intracerebral bleeding was the most common reason for withholding anticoagulation (37\%) at hospital dis-

Christian Gronemann and Stephan Hause contributed equally to this article. 
charge and stayed stable during the observation period. Conclusions: Changing national guidelines with discard of contraindications for anticoagulation and the introduction of DOACs led to a broader recommendation of oral anticoagulation. However, both, new guidelines and DOACs, were not found to be associated with an increasing percentage of patients discharged from our hospital already on recommended anticoagulant prevention. This might be explained by the decreasing length of hospital stay during the study period and a missing evidence of early bleeding risk of DOACs in patients with acute brain infarction. Evidencebased data to close this therapeutic gap are needed.

(C) 2020 The Author(s)

Published by S. Karger AG, Basel

\section{Introduction}

Oral anticoagulation is one of the most effective prevention of stroke recurrence in patients with atrial fibrillation (AF) comprising vitamin K antagonists (VKAs) [1] and direct oral anticoagulants (DOACs) [2-5]. Oral anticoagulation is, thus, widely recommended in stroke patients with AF by national and international guidelines [6-8]. This is in contrast to the results of national and international surveys among office-based physicians disclosing a sustained underuse of oral anticoagulation in those patients [9-12]. On the one hand, oral anticoagulation is mainly restrained to avoid major bleeding, which is an immanent side effect of any anticoagulant and considered an iatrogenic complication [13]. Ischemic stroke in patients not receiving anticoagulation, on the other hand, is deemed somewhat fateful.

Underuse or withhold of anticoagulation with VKAs has shown not only as a practicing doctors' phenomenon but also has been found in hospitalized patients with AF and high risk of stroke in a cross-sectional US study in the early 2000s, including all hospital categories [14]. However, the majority of acute stroke patients nowadays are treated in specialized stroke units with standardized diagnostic and therapeutic operating procedures [15], which may be responsible for shifting antithrombotic prevention in those patients toward anticoagulation [16].

We hypothesized that the introduction of DOACs reduced the risk of intracranial bleeding and, thus, making the handling of anticoagulation simpler than with VKAs. Change in German guidelines recommending anticoagulation in constellations earlier considered as contraindication [17] had influenced recommendation, prescription, and use of anticoagulation. To address these questions, we took into account of a large cohort of consecutive
Table 1. Demographics, baseline characteristics, antithrombotic premedication, and clinical findings in 1,812 patients with acute cerebral ischemia and $\mathrm{AF}$

\begin{tabular}{|c|c|}
\hline & $N(\%)$ \\
\hline Age, mean (SD), years & $76.7(9.6)$ \\
\hline Sex, male & $846(46.7)$ \\
\hline Hypertension & $1,711(94.4)$ \\
\hline Diabetes & $781(43.1)$ \\
\hline Hypercholesterolemia & $1,092(60.3)$ \\
\hline BMI, mean (SD), kg/m² & $27.7(5.4)$ \\
\hline History of myocardial infarction & $399(22.0)$ \\
\hline Congestive heart failure & $173(9.6)$ \\
\hline Peripheral vascular disease & $135(7.5)$ \\
\hline History of cerebral ischemia & $380(21.0)$ \\
\hline AF documented in medical history & $1,054(58.2)$ \\
\hline $\mathrm{CHADS}_{2}$ score $\geq 2$ by means of medical history & $1,503(82.9)$ \\
\hline \multicolumn{2}{|c|}{$\mathrm{CHA}_{2} \mathrm{DS}_{2} \mathrm{VASc}$ score $\geq 2$ by means of medical } \\
\hline history & $1,728(95.4)$ \\
\hline \multicolumn{2}{|l|}{ Antithrombotics at ischemic event } \\
\hline None & $649(35.9)$ \\
\hline Antiplatelets & $678(37.4)$ \\
\hline Anticoagulants & $485(26.8)$ \\
\hline NIHSS score at admission, median (IQR) & $7(3-16)$ \\
\hline \multicolumn{2}{|l|}{ Cerebral ischemia during } \\
\hline \multicolumn{2}{|l|}{ Current DGN guidelines version 5} \\
\hline Availability of DOAC (since Sep 2011) & $1,446(79.8)$ \\
\hline Hospital stay, days, median (IQR) & $9.5(5.9-15.0)$ \\
\hline MRS at discharge, median (IQR) & $3(2-4)$ \\
\hline \multicolumn{2}{|l|}{ Functional deficit at discharge/mortality } \\
\hline None/mild (mRS 0-2) & $754(41.6)$ \\
\hline Moderate/severe (mRS 3-5) & $939(51.8)$ \\
\hline Dead & $119(6.6)$ \\
\hline
\end{tabular}

$\mathrm{AF}$, atrial fibrillation; SD, standard deviation; IQR, inter quartile range; NIHSS, National Institutes of Health Stroke Scale; mRS, modified Rankin Scale; DGN, Deutsche Gesellschaft für Neurologie; DOAC, direct oral anticoagulant.

stroke patients with known or newly diagnosed AF admitted to our vascular and stroke center and investigated how the frequency of recommendation and prescription of anticoagulation together with affecting factors changed from 2009 to 2018 .

\section{Materials and Methods}

Patient Selection

Patients admitted to our stroke center with an acute ocular ischemic event, a transient ischemic attack, or an ischemic stroke underwent standard electrocardiography (ECG), 24-72-h ECG monitoring at the stroke unit, 24-h Holter monitoring during hospital stay, and assessment of medical history as part of a standard- 
ized diagnostic workup. Collected data were pseudonymized and entered into the institution's stroke registry. These registered patients were selected for the present analysis if AF had been revealed at ECG (monitoring) or documented in medical history.

\section{Data Collection and Study Objectives}

Registry data used for this analysis include results of vascular and cardiac diagnostics, including echocardiography and ECG/ ECG monitoring, collected comorbidities and vascular risk factors, neurological deficit at admission assessed by the National Institutes of Health Stroke Scale, functional outcome at discharge quantified by the modified Rankin Scale (mRS), calculated vascular risk scores $\mathrm{CHADS}_{2}$ and $\mathrm{CHA}_{2} \mathrm{DS}_{2} \mathrm{VASc}$, the antithrombotic drug used during the onset of the ischemic event leading to hospital admission, and prescribed antithrombotic prevention at discharge. Discharge letters of matching patients were collected and reviewed for recommended antithrombotic secondary prevention. An antithrombotic medication was considered as recommended by the treating physician if it either was recommended in the discharge letter or - in the absence of an explicit recommendation was prescribed at discharge. If the recommended antithrombotic prevention and that prescribed at discharge were different, the reason as presented in the discharge letter was assessed. Recommended and prescribed antithrombotic medication served as study objectives. If applicable, contraindications for anticoagulation according to the attending physician were collected from discharge letters and categorized into high bleeding risk (e.g., due to a high risk of falls), unfavorable benefit-to-risk ratio (i.e., persisting severe functional deficit without expected substantial worsening by a recurrent stroke, palliative/hospice care, and implanted left atrial occluder/prior atrial ablation), or other reasons.

\section{Statistics}

Statistical analysis was performed using the SPSS 25.0 software package. A possible linear trend of variables across observation years was investigated by conducting the $\chi^{2}$ test, one-way ANOVA, and Jonckheere-Terpstra test for grouped variables, parametric continuous variables, and nonparametric continuous variables, respectively. The impact of the observation year and of patients' demographics and clinical variables as listed in Table 1 on the study objectives (anticoagulation as recommended secondary prevention in discharge letter; anticoagulant use at discharge from hospital) were estimated by binary logistic regression analysis with backward stepwise removal $(p>0.10)$ and entry selection $(p<0.05)$. Confidence interval (CI) was set at $95 \%$ and significance at a $p$ value of less than 0.05 for two-sided tests.

\section{Results}

From January 2009 to December 2018, 7,175 consecutive patients suffering from acute ischemic event were admitted to our stroke center. Out of these, 1,812 patients (25.3\%) met the inclusion criterion of AF documented in medical history $(1,054 ; 58.2 \%$ of AF patients) or detected for the first time on ECG (758; 41.8\%) (Fig. 1). Data of those 1,812 cases comprising patients' demographics,

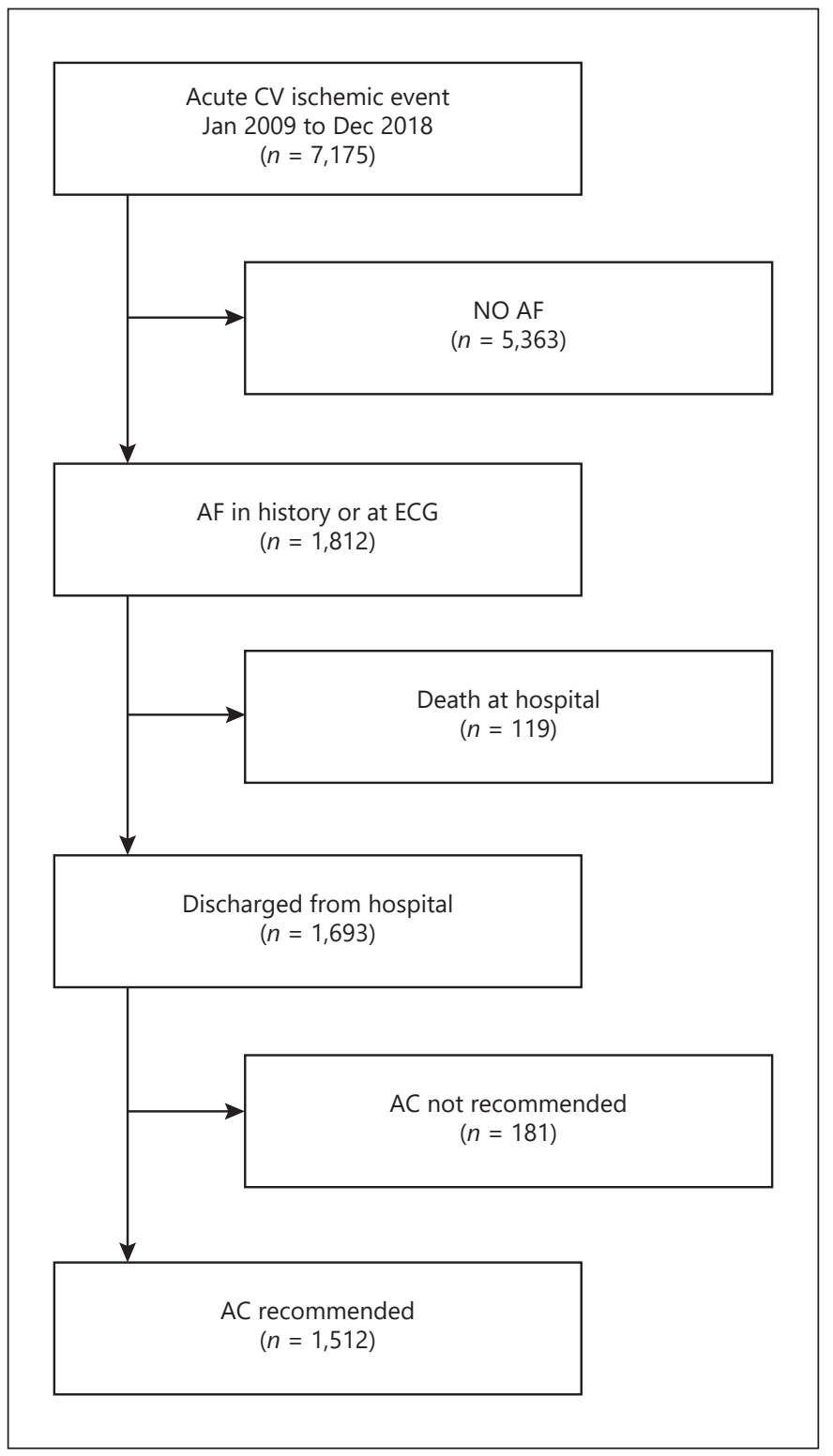

Fig. 1. Patients' selection. $\mathrm{CV}$, cerebrovascular; $\mathrm{AF}$, atrial fibrillation; ECG, electrocardiography, AC, anticoagulation.

baseline characteristics, antithrombotic premedication, and clinical findings are presented in Table 1. Fraction of patients with hypercholesterolemia (43.4-72.1\%; $p<$ 0.001 ) increased within the whole observation period. We consider this due to a shift in diagnostic criteria driven through lower threshold for this diagnosis and due to a stricter treatment, especially in high-risk patients with ischemic events. Mean age (77.0-77.8 years; $p=0.002)$, AF already documented in medical history (51.2-66.2\%; 0.039 ), and a pre-stroke $\mathrm{CHADS}_{2}$ score of at least 2 (76.7- 


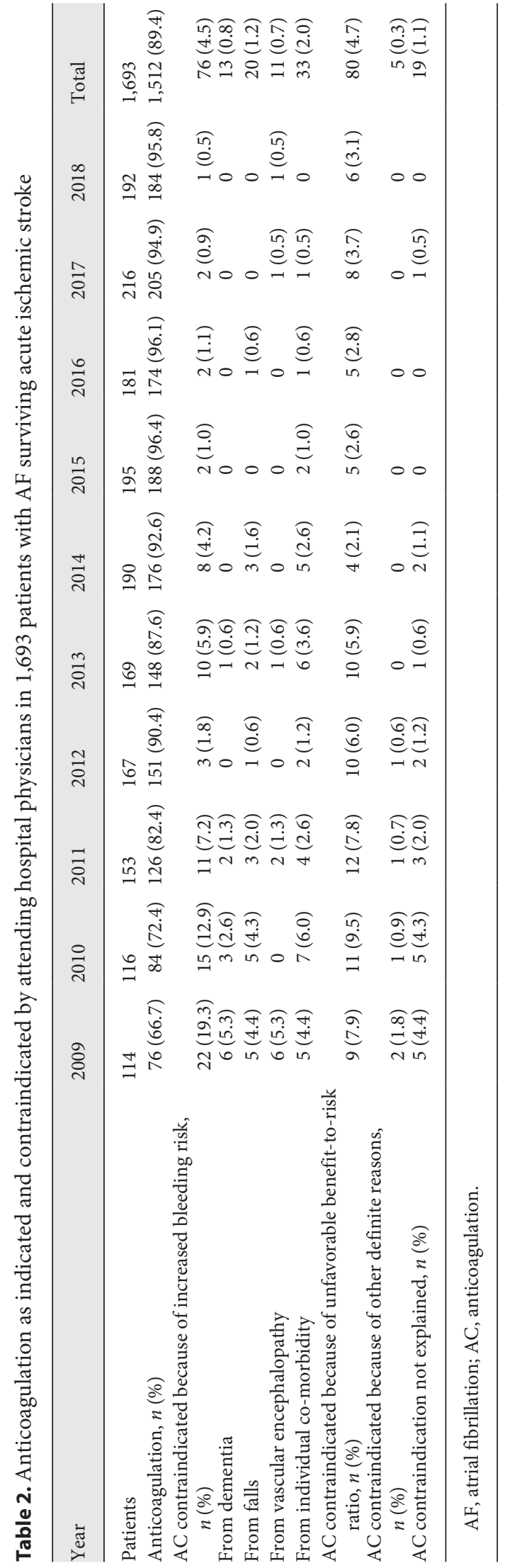

Modified In-Hospital Stroke Prevention with DOACs

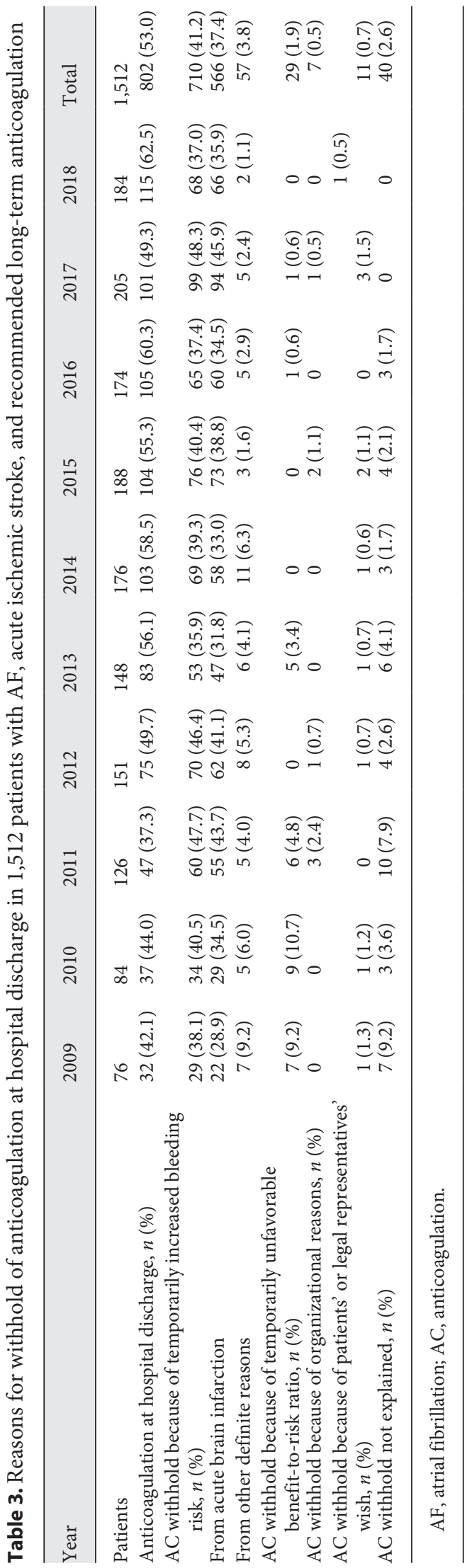


Fig. 2. Percentage of patients with already prescribed anticoagulants and with still only recommended anticoagulation at discharge from hospital in 1,512 AF patients with an acute ischemic event and recommended anticoagulation by the treating physician. AF, atrial fibrillation; DOAC, direct oral anticoagulant; VKA, vitamin $\mathrm{K}$ antagonist; LMWH, low molecular weight heparin.

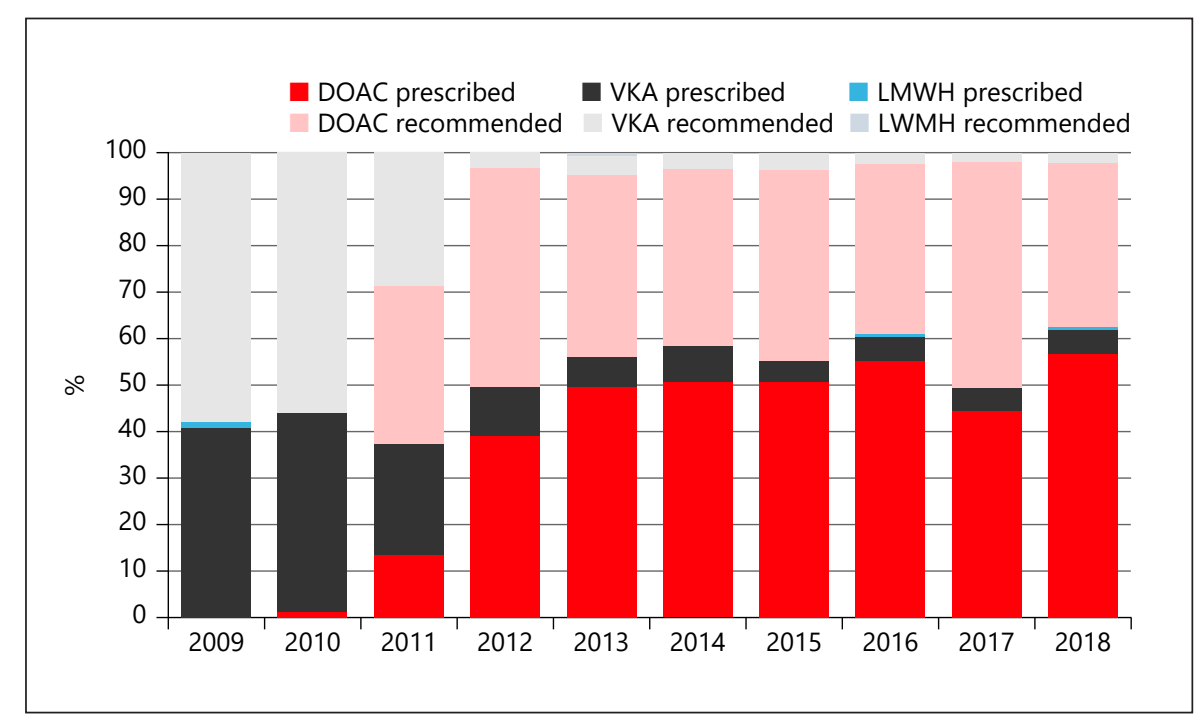

83.6\%; $p=0.021)$ increased within the observation period and median length of hospital stay decreased (12.7-8.9 days; $p<0.001)$. Significant changes $(p<0.001)$ were also observed in the patients' antithrombotic premedication at admission with an increase of part of patients on oral anticoagulation (12.4-41.8\%) and a decrease of those on antiplatelet agents (46.5-28.4\%) and without antithrombotic premedication (41.1-29.8\%).

One hundred nineteen patients $(6.6 \%)$ died during the hospital stay leaving 1,693 surviving patients discharged from hospital (93.4\%) who underwent further analysis. Annual frequency of anticoagulation for secondary prevention as indicated by the attending physicians in those 1,693 surviving patients is presented in Table 2. Percentage of patients with indicated oral anticoagulation increased within the observation period from 66.7 to $95.8 \%$ (per year; adjusted odds ratio [OR] 1.309; CI 1.1531.486). Vice versa, we found a simultaneous decrease of those in whom oral anticoagulation was considered contraindicated because of bleeding risk, unfavorable benefit-to-risk ratio, or other reasons such as patients will. DOAC approval (adjusted OR, 4.026; CI, 1.962-8.265) and changing guidelines (adjusted OR, 2.184; CI, 1.0064.743) were also associated with an increasing indication for patients' anticoagulation independently from time trend and each other. A reverse effect, that is, less frequent indication for oral anticoagulation was found for patients with an increasing $\mathrm{mRS}$ score at discharge (per point; adjusted OR, 0.538; CI, 0.469-0.617), increasing age (per year; adjusted OR, 0.936; CI, 0.911-0.963), and male sex (adjusted OR, 0.676; CI, 0.466-0.982). Since 2014, patients not recommended any anticoagulation because of an increased bleeding risk either had a combination of high risk of fall and at least one other relative contraindication (cerebral amyloid angiopathy in 2 patients and refractory epilepsy in 1) or they could not be treated with DOACs due to pronounced renal insufficiency and did not show enough compliance for VKA therapy (3 patients).

The fraction of patients receiving recommended anticoagulation for secondary prevention already at discharge from hospital increased within the observation period from 42.1 to $62.5 \%$ (per year; adjusted OR, 1.079; CI 1.004-1.160) (Table 3). Although the introduction of DOACs resulted in a broad replacement of VKAs (Fig. 2), it was not associated independently with an increased prescription rate of anticoagulants at patients' discharge from hospital in multivariate analysis. Higher rates of prescription of anticoagulation could be demonstrated for a longer hospital stay (per day; adjusted OR, 1.044; CI 1.028-1.055) and a lower mRS score at discharge (per point; adjusted OR, 0.472; CI, 0.432-0.516). Severe stroke with larger infarcts led to later recommended point of time to start anticoagulation, which we did not register as prescribed in this study when patients went to rehabilitation hospital. Surprisingly, guideline change 2012 was associated with a decreased rate of anticoagulation at hospital discharge (adjusted OR, 0.641; CI, 0.414-0.991). It is remarkable that fear of intracerebral bleeding into acute brain infarction was the most frequent cause to withhold anticoagulation at patients' discharge from hospital (37.4\%), which remained stable during the observed time period (Table 3 ), and was not influenced by DOAC introduction and the guideline change. 


\section{Discussion}

Between 2009 and 2018, the proportion of patients with AF for whom the treating physicians indicated anticoagulation after a stroke increased significantly. Whereas we recommended anticoagulation in merely two-thirds of patients in 2009, this rate increased up to $95 \%$ in 2018. Our study suggests that both the introduction of DOACs in September 2011 and the revision and publication of the corresponding stroke guideline in August 2012 independently and distinct from a possible general time trend contributed to this trend, DOAC introduction probably by promising a lower cerebral bleeding risk compared to VKAs and guideline revision by relativizing or abandoning previous general contraindications like dementia, risk of fall, and vascular encephalopathy $[6,17]$. As a consequence, since 2014, only 6 patients had been considered having one of those contraindications (Table 2). Moreover, combined with an older age and a more severe deficit at discharge, those contraindications also may have been the driving reasons for an unfavorable benefit-to-risk ratio as the other prominent reason for anticoagulation withhold (Table 2). This is supported by the fact that since 2014, an unfavorable benefitto-risk ratio was considered only in patients receiving palliative care, whereas the years before a contraindication was estimated even in patients assumed remaining permanently bedridden.

Number of patients who took the recommended anticoagulation at discharge from hospital increased from 42.1 to $62.5 \%$ during the observation period. At the same time, the percentage of patients for whom anticoagulation was considered (still) contraindicated at discharge decreased for all contraindications except for those patients with a temporary increased risk of bleeding into an acute infarct or other temporary increased bleeding risk (e.g., newly diagnosed and, therefore, untreated macro-uremia or gastrointestinal bleedings). The fear of early bleeding complications might result predominantly from missing early DOAC application data in the pivotal trials [2-5], which only allowed start of anticoagulation after 10-30 days after the ischemic event. This may be enhanced by a decreasing hospital stay from within the observation period, which was an independent predictor to withhold recommended anticoagulation at discharge. We could not find other confounders restraining us from earlier anticoagulation with DOACs, especially not the severity of stroke, which did not change during the observation period. Consequently, the increased numbers of patients already anticoagulated at discharge was not found to be associated with the introduction of DOACs and may be driven due to broader indication of anticoagulation.
Further trials addressing those questions about timing the (re-)start of anticoagulation with respect to the risk of early bleeding complications are required. PRODAST (Prospective Record Of the use of Dabigatran in patients with Acute Stroke or TIA, NCT02507856) is an example of them [18].

We found a negative impact of the change in guidelines on the number of patients' anticoagulated at discharge in our multivariate analysis. There can only be speculation about the reason for this. We recommended oral anticoagulation more generously but might restrain the onset of medication due to fear of early infarct bleeding.

Even though a number of overall 1,693 patients empowering our study, this is a single-center study and, therefore, limited by possible treatment bias. However, in case of missing evidence, the start of anticoagulation after an ischemic stroke was in the decision of the attending senior physician without department's directive. In addition, a selection bias could not be excluded as this study was conducted on a stroke center, which might result in a cohort of more impaired patients with larger infarcts compared to other hospitals.

As a university center, we actively followed the reasons and considerations resulting in guideline change and might have anticipated some effects. On the other hand, even in the presence of new guidelines, implementation of SOP may have required some time. Neither the effect of the former nor the latter could be estimated and, hence, the date of administrative guideline changes was used as cutoff for the introduction of new guidelines at statistical analyses.

\section{Conclusion}

Discarding the contraindications in revised guidelines and the introduction of DOACs led to a broader recommendation of oral anticoagulation. Even though DOACs are commonly known for fewer intracerebral bleeding, missing evidence of bleeding risk in patients with acute brain infarction and decreasing length of hospital stay hindered prescription of recommended anticoagulation already at hospital discharge in a substantial number of patients. Evidence-based data to close this therapeutic gap are needed.

\section{Acknowledgement}

We are grateful for the support of our stroke center staff. 


\section{Statement of Ethics}

Registry storing and scientific evaluation of pseudonymized data obtained in routine clinical practice as well as scientific evaluation of clinical discharge letters were performed in accordance with European, national, and federal data protection laws so that the responsible ethics committee of the University of Magdeburg waived the need for patient's consent.

\section{Conflict of Interest Statement}

The authors have no conflicts of interest to declare.

\section{Funding Sources}

The authors did not receive any funding.

\section{Author Contributions}

M.G. planed and initiated this study, collected patients' data by means of pseudonymized register data and discharge letters. C.G. (from 2009 until 2015) and S.H. (from 2015 until 2018) reviewed the letters for recommended antithrombotic secondary prevention. S.H., C.G., and M.G. wrote the first draft of the manuscript. A.A., J.N., S.S., and H.H. contributed ideas and edited the final draft. All authors read and approved the final manuscript.

\section{References}

1 Group EEAFTS. Secondary prevention in non-rheumatic atrial fibrillation after transient ischaemic attack or minor stroke. Lancet. 1993;342:1255-62.

2 Connolly SJ, Ezekowitz MD, Yusuf S, Eikelboom J, Oldgren J, Parekh A, et al. Dabigatran versus warfarin in patients with atrial fibrillation. N Engl J Med. 2009;361(12):1139-51.

3 Giugliano RP, Ruff CT, Braunwald E, Murphy SA, Wiviott SD, Halperin JL, et al. Edoxaban versus warfarin in patients with atrial fibrillation. N Engl J Med. 2013;369(22):2093-104.

4 Granger CB, Alexander JH, McMurray JJ, Lopes RD, Hylek EM, Hanna M, et al. Apixaban versus warfarin in patients with atrial fibrillation. N Engl J Med. 2011;365(11):98192.

5 Patel MR, Mahaffey KW, Garg J, Pan G, Singer DE, Hacke W, et al. Rivaroxaban versus warfarin in nonvalvular atrial fibrillation. $\mathrm{N}$ Engl J Med. 2011;365(10):883-91.

6 Diener HC, Weimar C, Berlit P, Deuschl G, Elger C, Gold R, et al., editors. Leitlinien für diagnostik und therapie in der neurologie. 5th fully reviced ed. Stuttgart, New York, Delhi, Rio: Thieme Verlagsgruppe; 2012.

7 January CT, Wann LS, Alpert JS, Calkins H, Cigarroa JE, Cleveland JC Jr, et al. 2014 AHA/
ACC/HRS guideline for the management of patients with atrial fibrillation: a report of the American College of Cardiology/American Heart Association Task Force on Practice Guidelines and the Heart Rhythm Society. J Am Coll Cardiol. 2014;64(21):e1-76.

8 Kirchhof P, Benussi S, Kotecha D, Ahlsson A, Atar D, Casadei B, et al. 2016 ESC Guidelines for the management of atrial fibrillation developed in collaboration with EACTS. Eur Heart J. 2016;37(38):2893-962.

9 Barnes GD, Lucas E, Alexander GC, Goldberger ZD. National trends in ambulatory oral anticoagulant use. Am J Med. 2015; 128(12):1300-5 e2.

10 Camm AJ, Accetta G, Ambrosio G, Atar D, Bassand JP, Berge E, et al. Evolving antithrombotic treatment patterns for patients with newly diagnosed atrial fibrillation. Heart. 2017;103(4):307-14.

11 Kirley K, Qato DM, Kornfield R, Stafford RS, Alexander GC. National trends in oral anticoagulant use in the United States, 2007 to 2011. Circ Cardiovasc Qual Outcomes. 2012;5(5) 615-21.

12 Kip M, Schönfelder T, Bleß HH. Weißbuch schlaganfallprävention bei vorhofflimmern. 1st ed. Stuttgart: Thieme-Verlag; 2015.
13 Wehbe RM, Yadlapati A. Underuse of oral anticoagulants for nonvalvular atrial fibrillation: past, present, and future. Tex Heart Inst J. 2016;43(4):287-90.

14 Waldo AL, Becker RC, Tapson VF, Colgan KJ. Hospitalized patients with atrial fibrillation and a high risk of stroke are not being provided with adequate anticoagulation. J Am Coll Cardiol. 2005;46(9):1729-36.

15 Aguiar de Sousa D, von Martial R, Abilleira S, Gattringer T, Kobayashi A, Gallofre M, et al. Access to and delivery of acute ischaemic stroke treatments: a survey of national scientific societies and stroke experts in 44 European countries. Eur Stroke J. 2019;4(1):1328.

16 Wutzler A, Krogias C, Grau A, Veltkamp R, Heuschmann PU, Haeusler KG. Stroke prevention in patients with acute ischemic stroke and atrial fibrillation in Germany: a cross sectional survey. BMC Neurol. 2019;19(1):25.

17 Diener HC, LdDu DSG. Primär- und Sekundärprävention der zerebralen Ischämie. Leitlinien der DGN 2008. Stuttgart: ThiemeVerlag; 2008.

18 Diener HC, Weimar C, Stang A. PRODAST: prospective record of the use of dabigatran in patients with acute stroke or TIA2018. 2018 